\title{
OTIMIZAÇÃO DA PROGRAMAÇÃO DAS LIMPEZAS EM UMA BATERIA DE PRÉ-AQUECIMENTO CONSIDERANDO A VARIAÇÃO DA TAXA DE DEPOSIÇÃO EM FUNÇÃO DA TEMPERATURA
}

\author{
J. C. $\operatorname{LEMOS}^{1}$, E. M. QUEIROZ ${ }^{2}$, A. L. H. $\operatorname{COSTA}^{1}$ \\ ${ }^{1}$ Universidade do Estado do Rio de Janeiro, \\ ${ }^{2}$ Universidade Federal do Rio de Janeiro, \\ E-mail para contato: andrehc@uerj.br
}

\begin{abstract}
RESUMO - Na literatura, há diversos trabalhos que investigam a otimização das paradas para limpeza dos trocadores de calor presentes nas unidades de destilação atmosférica de refinarias, visando minimizar o consumo de energia. Entretanto, estes trabalhos utilizam modelos de deposição simplificados, que não levam em conta a variação da taxa de deposição em função das condições operacionais. Buscando contornar esta limitação, o presente trabalho propõe uma abordagem de otimização baseada na solução de uma sequência de problemas de programação linear inteira mista, onde a taxa de deposição é atualizada considerando o modelo de EbertPanchal modificado. Cada um dos problemas indica quais trocadores devem ser limpos em um determinado instante de tempo, de tal forma que a combinação de seus resultados compõe a solução final da programação das limpezas. A aplicabilidade da metodologia proposta é ilustrada através de um exemplo típico de uma bateria de pré-aquecimento de uma refinaria.
\end{abstract}

\section{INTRODUÇÃO}

A deposição em redes de trocadores de calor é um problema industrial recorrente, pois diminui o coeficiente global de troca térmica, através do surgimento de resistências térmicas adicionais devido ao acúmulo de depósitos. Um importante exemplo deste efeito ocorre no processo de refino do petróleo. Na destilação do petróleo, a corrente de óleo cru deve ser aquecida até aproximadamente $380^{\circ} \mathrm{C}$ para ser alimentada na torre de destilação. Uma rede de trocadores de calor, denominada bateria de pré-aquecimento, é utilizada para reduzir o consumo de energia necessária para aquecer o petróleo através da troca de calor deste com as correntes laterais da torre de destilação. Infelizmente, estes trocadores de calor que compõem a bateria de pré-aquecimento estão sujeitos à deposição, que com o tempo aumenta o consumo de combustível no forno, o que acarreta efeitos prejudiciais econômica e ambientalmente.

Para diminuir a perda de recuperação de energia nesta bateria, devido ao efeito da deposição, os trocadores de calor devem ser limpos periodicamente. A identificação da melhor programação destas limpezas é um problema complexo, pois deve considerar o ganho futuro ao se realizar uma limpeza e, 


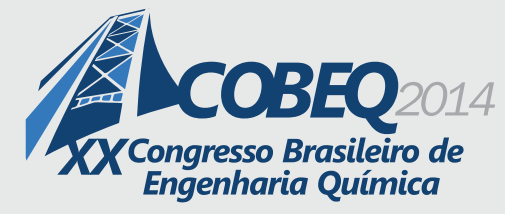

também, o aumento no consumo de utilidades que se deve ao fato do trocador estar off-line durante a limpeza, diminuindo a recuperação de energia naquele momento.

Devido à importância deste problema, diversos trabalhos foram desenvolvidos utilizando diferentes abordagens para identificar a programação ótima das limpezas em uma bateria de préaquecimento. Por exemplo, no âmbito da aplicação de técnicas de programação matemática, Smaïli et al. (2001) e Lavaja e Bagajewicz (2004) resolveram o problema como um problema de programação não-linear inteira mista (MINLP) e programação linear inteira mista (MILP), respectivamente. Um aspecto comum a estes trabalhos corresponde à utilização de modelos empíricos de taxa de deposição, onde a taxa não depende das condições operacionais. Entretanto, ao ignorar este aspecto fenomenológico do problema, é introduzido um afastamento potencialmente importante em relação à realidade, o que pode comprometer a qualidade da solução.

Visando contribuir para preencher esta lacuna, o presente trabalho propõe o emprego de uma técnica do tipo horizonte móvel, baseada na solução de uma série de problemas MILP, onde em cada instante a abordagem desenvolvida permite a seleção dos melhores trocadores de calor a serem limpos. A taxa de deposição empregada em cada problema MILP resolvido é atualizada através do modelo de Ebert Panchal modificado, proposto por Panchal et al. (1997). Desta forma, as modificações da taxa de deposição resultantes da alteração do perfil de temperatura acarretadas pelas limpezas são contempladas na solução ótima encontrada.

\section{ESQUEMA DE OTIMIZAÇÃO}

O conjunto de trocadores que deve ser limpo em um determinado instante de tempo, considerando o efeito destas limpezas em um horizonte móvel, pode ser determinado através da solução de um problema MILP proposto por Assis et al. (2012). A extensão deste modelo no contexto de horizonte móvel empregada aqui foi desenvolvida em Lemos et al. (2013), embora com taxas de deposição constantes.

Neste trabalho, a programação ótima proposta é dada pela combinação dos resultados para cada instante de tempo, ou seja, o resultado de cada problema MILP, de tal maneira que, para o instante seguinte, as resistências de depósito são atualizadas de acordo com as decisões de limpeza ou não através do modelo Ebert Panchal modificado. A Figura 1 representa a abordagem proposta, onde os quadrados pretos representam uma limpeza.

\subsection{Estrutura da Rede}

A bateria de pré-aquecimento é representada por um dígrafo, onde as arestas $k$ correspondem as correntes de processo $(k \in S T R)$, e os vértices $t$, aos elementos da rede $(t \in V E T)$. As correntes de processo estão divididas em correntes quentes $(H S T R)$ e correntes frias (CSTR). Os elementos da rede estão divididos em trocadores de calor $(H E)$, misturadores $(M X)$, divisores $(S P)$, dessalgadoras $(D S)$, unidades de suprimento $(P S)$ e unidades de demanda $(P D)$. As unidades de suprimento e demanda representam as correntes de entrada e saída da rede. 


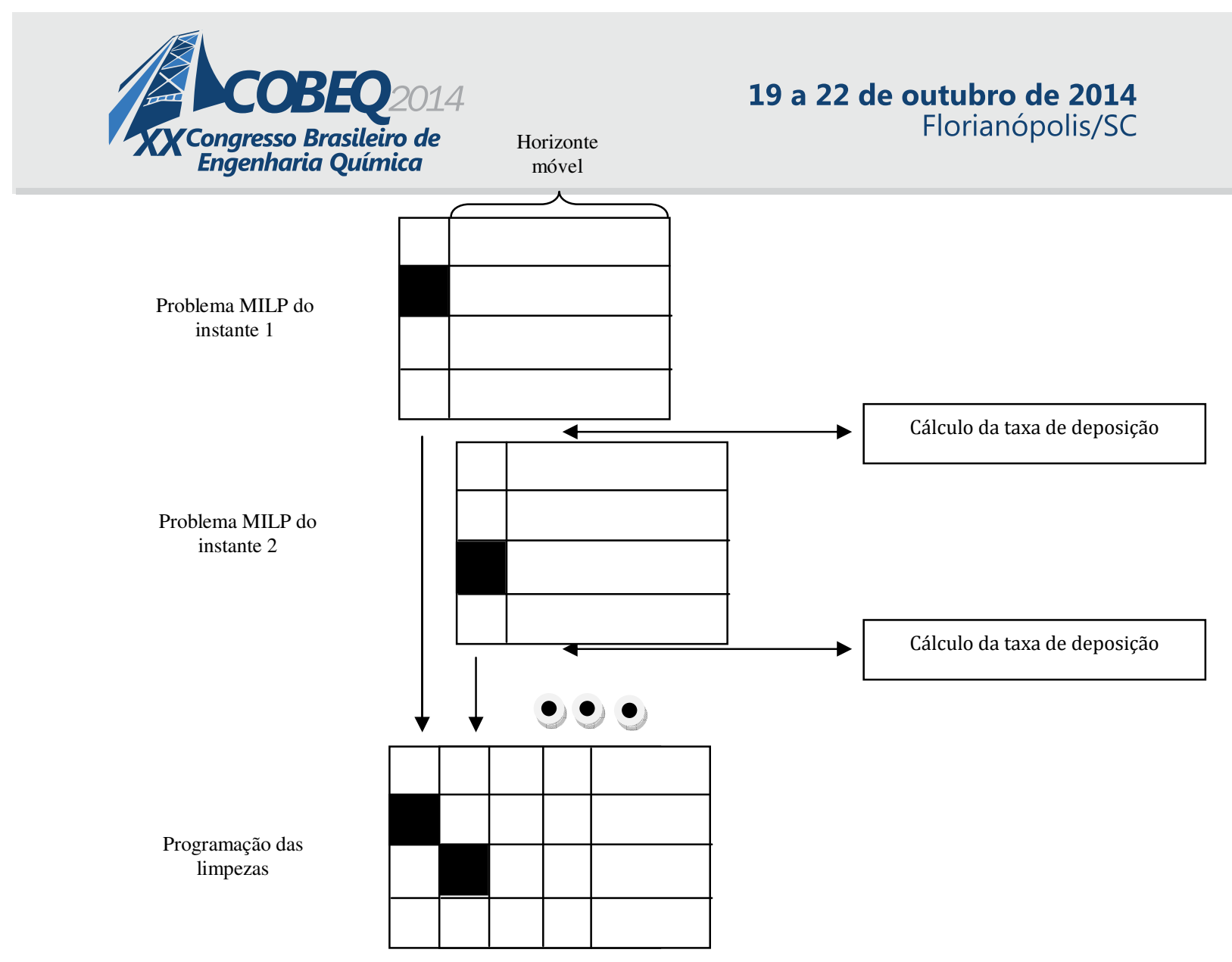

Figura 1 - Esquema da otimização

\subsection{Variáveis}

No problema em questão, existem variáveis contínuas e binárias. As variáveis contínuas são as temperaturas e as cargas térmicas, e as variáveis binárias indicam quais trocadores devem ser limpos para um instante de tempo. Cada instante de tempo é identificado pelo índice $\tau(\tau \in T I)$.

A variável $T_{k, \tau}$ representa a temperatura da corrente de processo $k$ no instante $\tau$. A variável $V_{t, \tau}$ representa a temperatura de entrada/saída do vértice de suprimento/demanda $t$ no instante $\tau$. A carga térmica do trocador de calor $t$ no instante $\tau$ é representada por $Q_{t, \tau}$. A variável binária $y_{t}$ indica se o trocador de calor $t$ deve ser limpo (=1) ou não (=0). As vazões mássicas das correntes de processo, $m_{k}$, e as vazões mássicas nos vértices de suprimento/demanda, $n_{t}$, são conhecidas, ou seja, são parâmetros para o problema de otimização.

\subsection{Função Objetivo}

A função objetivo considera a integração dos custos das utilidades, durante um horizonte curto a frente do instante de tempo analisado, somado aos custos de limpeza:

$$
f o b j=\sum_{t \in P D} \sum_{\tau \in T I} p_{\tau} s_{\tau} C_{O P, t} n_{t} C P_{t}\left(V_{r e f, t}-V_{t, \tau}\right)+\sum_{t \in H E} y_{t} C_{C, t}+C_{\mathrm{int}}
$$

onde $p_{\tau}$ são os pesos do método de integração numérica utilizado (método do trapézio), $s_{\tau}$ são os 
fatores para valor presente, $C_{O P, t}$ é o custo das utilidades associado à corrente de saída do vértice $t, n_{t}$ e $V_{t}$ são a vazão e temperatura associadas ao vértice $t$, ref é o subscrito que indica a temperatura de saída da rede, $C_{C, t}$ são os custos de limpeza e $C_{i n t}$ é o somatório do gasto de energia nos instantes anteriores, cuja programação ótima já foi identificada.

\subsection{Restrições - Balanços de Energia}

Os balanços de energia nos vértices da rede são dados por:

$$
\begin{array}{lr}
\sum_{k \in S_{t}^{\text {in }}} m_{k} C p_{k} T_{k, \tau}-\sum_{k \in S_{t}^{\text {out }}} m_{k} C p_{k} T_{k, \tau}=0 & t \in(M X \cup S P), \tau \in T I \\
\sum_{k \in S_{t}^{\text {sin }}} m_{k} C p_{k} T_{k, \tau}-\sum_{k \in S_{t}^{\text {out }}} m_{k} C p_{k} T_{k, \tau}+n_{t} C P_{t} V_{t, \tau}=0 & t \in(P S \cup P D), \tau \in T I \\
\sum_{\left.k \in S_{t}^{\text {in }} \cap H S T R\right)} m_{k} C p_{k} T_{k, \tau}-\sum_{k \in\left(S_{t}^{\text {out }} \cap H S T R\right)} m_{k} C p_{k} T_{k, \tau}-Q_{t, \tau}=0 & t \in H E, \tau \in T I \\
\sum_{\left.k \in S_{t}^{\text {in }} \cap C S T R\right)} m_{k} C p_{k} T_{k, \tau}-\sum_{k \in\left(S_{t}^{\text {out }} \cap C S T R\right)} m_{k} C p_{k} T_{k, \tau}+Q_{t, \tau}=0 & t \in H E, \tau \in T I \\
T_{k, \tau}-T_{k^{\prime}, \tau}=\Delta_{t} & t \in D S, k \in S_{t}^{\text {in }}, k^{\prime} \in S_{t}^{\text {out }}, \tau \in T I \\
T_{k, \tau}-T_{k^{\prime}, \tau}=0 & t \in S P, k \in S_{t}^{\text {in }}, k^{\prime} \in S_{t}^{\text {out }}, \tau \in T I \\
V_{t, \tau}-V_{t, \tau}^{\text {spe }}=0 & t \in P S, \tau \in T I
\end{array}
$$

onde $C p_{k}$ é a capacidade calorífica da corrente $k, C P_{t}$ é a capacidade calorífica da corrente que entra/sai pelo vértice de suprimento/demanda $t, \Delta_{t}$ é a variação de temperatura na dessalgadora $t$, o sobrescrito spe identifica as temperaturas especificadas dos vértices de suprimento da rede, e os sobrescritos in e out identificam o conjunto de arestas que entram/saem do vértice $t$.

\subsection{Restrições - Equações dos trocadores de calor}

O comportamento no estado estacionário dos trocadores de calor da rede é descrito pelo método P-NUT. A efetividade em cada trocador ao longo do horizonte móvel é calculada previamente considerando duas alternativas: o trocador foi limpo $\left(P^{\text {yes }}\right)$ ou não $\left(P^{n o}\right)$.

Após uma manipulação adequada, capaz de substituir eventuais termos bilineares por desigualdades lineares correspondentes, as equações dos trocadores de calor assumem a seguinte forma:

$$
T_{k, \tau}-U T\left(1-y_{t}\right) \leq y h_{t, \tau} \leq T_{k, \tau}-L T\left(1-y_{t}\right) \quad t \in H E, \tau \in T I, k \in\left(H S T R \cap S_{t}^{i n}\right)
$$




$$
\begin{aligned}
& T_{k, \tau}-U T\left(1-y_{t}\right) \leq y c_{t, \tau} \leq T_{k, \tau}-L T\left(1-y_{t}\right) \quad t \in H E, \tau \in T I, k \in\left(C S T R \cap S_{t}^{i n}\right) \\
& L T y_{t} \leq y h_{t, \tau} \leq U T y_{t} \quad t \in H E, \tau \in T I \\
& L T y_{t} \leq y c_{t, \tau} \leq U T y_{t} \quad t \in H E, \tau \in T I \\
& P_{t, \tau}^{n o} T_{k, \tau}+\left(1-P_{t, \tau}^{n o}\right) T_{k^{\prime}, \tau}-T_{k^{\prime \prime}, \tau}-y h_{t, \tau}\left(P_{t, \tau}^{y e s}-P_{t, \tau}^{n o}\right)+y c_{t, \tau}\left(P_{t, \tau}^{y e s}-P_{t, \tau}^{n o}\right)=0 \\
& t \in H E, \tau \in T I, k \in\left(C S T R \cap S_{t}^{i n}\right), \quad k^{\prime} \in\left(H S T R \cap S_{t}^{i n}\right), k^{\prime \prime} \in\left(H S T R \cap S_{t}^{o u t}\right)
\end{aligned}
$$

Os valores de efetividade presentes nas equações acima são determinados baseados em valores de taxa de deposição oriundos do modelo Ebert-Panchal modificado:

$$
\frac{d R_{f}}{d t}=\alpha R e^{-0,66} \operatorname{Pr}^{-0,33} \exp \left(-\frac{E_{a}}{R T_{f i m}}\right)-\gamma \tau_{w}
$$

onde $R e$ é o número de Reynolds, $P r$ é o número de Prandtl, $T_{f i l m}$ é a temperatura de filme e $\tau_{w}$ é a tensão de cisalhamento.

\subsection{Restrições - Limitações dos recursos}

O número máximo de limpezas em um instante de tempo é indicado pela seguinte equação:

$$
\sum_{t \in H E} y_{t}-N_{\max } \leq 0
$$

\section{RESULTADOS}

A abordagem proposta foi analisada através de sua aplicação em um exemplo genérico de uma rede de trocadores de calor apresentada na Figura 2.

A corrente fria de alimentação possui vazão $41,7 \mathrm{~kg} / \mathrm{s}$, inicialmente a uma temperatura de 100 ${ }^{\circ} \mathrm{C}$. As propriedades físicas correspondentes, assumidas constantes, são: $\rho=818,8 \mathrm{~kg} / \mathrm{m}^{3} ; \mathrm{Cp}=2400$ $\mathrm{J} / \mathrm{kgK}, \mu=1,27.10^{-3} ; \mathrm{k}=0,10 \mathrm{~W} / \mathrm{mK}$. As correntes quentes de entrada na rede possuem vazão de 17,8 $\mathrm{kg} / \mathrm{s}$ com $\mathrm{Cp}=2800 \mathrm{~J} / \mathrm{kgK}$. As temperaturas de entrada correspondentes são $250,0{ }^{\circ} \mathrm{C}$ e $350{ }^{\circ} \mathrm{C}$, respectivamente. Todos os trocadores de calor presentes na rede são do tipo casco-e-tubo com 1 passe no casco e 4 nos tubos, área de troca térmica de $800 \mathrm{~m}^{2}$, com o fluido frio escoando no lado dos tubos. Os tubos dos trocadores de calor possuem um diâmetro externo de $19,05 \mathrm{~mm}$, a resistência condutiva nos tubos foi desprezada. Os coeficientes de transferência de calor por convecção do lado dos tubos e do lado do casco são iguais a $400 \mathrm{~W} /\left(\mathrm{m}^{2} \mathrm{~K}\right)$ e $400 \mathrm{~W} /\left(\mathrm{m}^{2} \mathrm{~K}\right)$, respectivamente. Assume-se que os trocadores estão inicialmente limpos. 


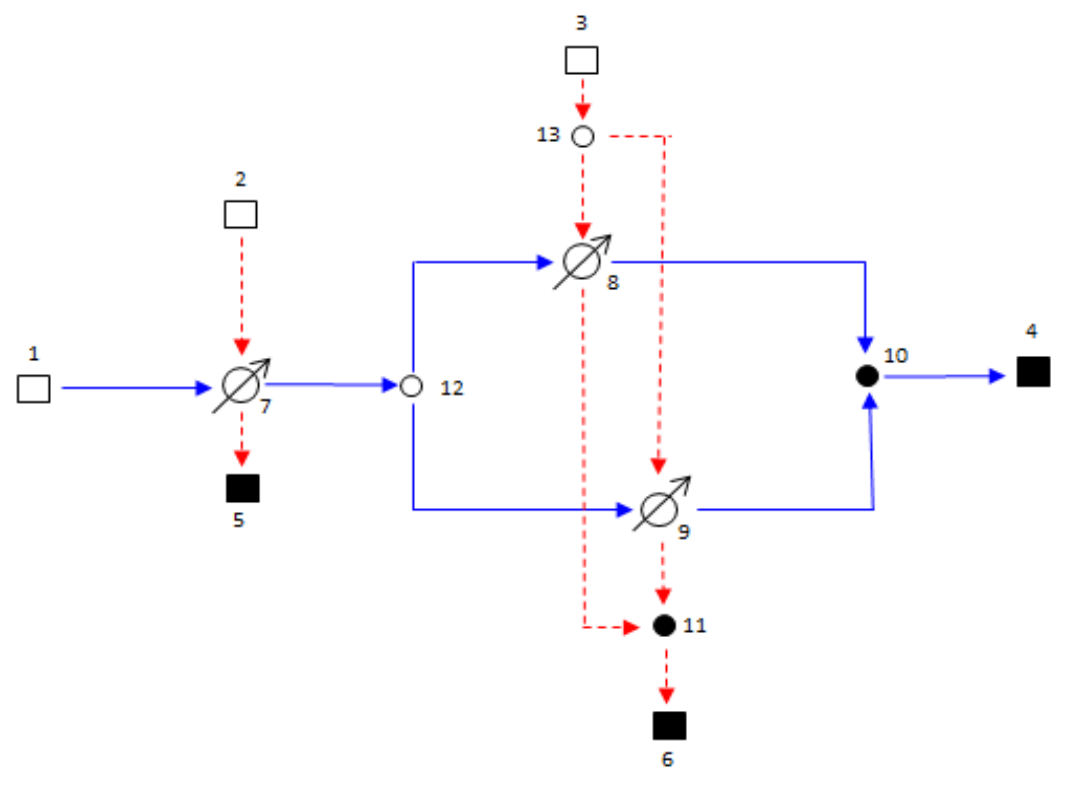

Figura 2 - Fluxograma da rede de trocadores de calor

O horizonte total considerado é de 2 ano, com o tempo discretizado em períodos de uma semana. Para a função objetivo foi utilizada uma temperatura de referência de $255,9^{\circ} \mathrm{C}$, que representa a temperatura de saída da rede se todos os trocadores de calor estivessem limpos. O custo da energia foi considerado $3,7 \cdot 10^{-3} \$ / \mathrm{MJ}$, o custo da limpeza zero, e a taxa de atratividade igual a $0 \%$. Assumiu-se ainda que até 2 trocadores possam ser limpos simultaneamente. O horizonte de tempo móvel considerado envolve 8 semanas. Os parâmetros adotados para o modelo de Ebert-Panchal modificado foram: $\alpha=127,7 \mathrm{~m}^{2} \mathrm{~K} / \mathrm{J} ; \gamma=3,44.10^{15} \mathrm{~m}^{2} \mathrm{~K} / \mathrm{J}$ Pa $e E_{a}=75000 \mathrm{~J} / \mathrm{mol}$.

Para efeito de comparação, os resultados encontrados através da metodologia proposta são apresentados na Tabela 1 juntamente com o desempenho da rede sem limpezas e o desempenho correspondente se a programação das paradas tivesse sido otimizada com taxas de deposição constantes (abordagem tradicional via modelo puramente linear).

Tabela 1 - Função objetivo e ganho

\begin{tabular}{|c|c|c|}
\hline Abordagem & Função objetivo & Ganho (\%) \\
\hline Sem limpezas & 0,66 & 0 \\
\hline Otimização proposta & 0,341 & 48,25 \\
\hline Otimização tradicional & 0,363 & 44,92 \\
\hline
\end{tabular}

Como se pode observar na Tabela 1, a adoção da programação das limpezas é capas de trazer ganhos consideráveis. Neste sentido, a adoção de um modelo fenomenológico para atualização dos 
valores de taxa, se mostra superior à abordagem tradicional. Sua superioridade pode não parecer muito significativa, mas se tratando de grandes redes esta diferença de ganho pode representar valores financeiramente significativos.

A programação ótima das limpezas encontra-se apresentada na Tabela 2 e os perfis da taxa de deposição nos trocadores da rede podem ser visualizados na Figura 3.

Tabela 2 - Programação ótima das limpezas

\begin{tabular}{|c|c|c|c|c|c|c|c|}
\hline Trocador & \multicolumn{7}{|c|}{ Tempo } \\
\hline & 11 & 23 & 35 & 47 & 59 & 72 & 85 \\
\hline 7 & & & & & & & \\
\hline 8 & & & & & & & \\
\hline 9 & & & & & & & \\
\hline
\end{tabular}

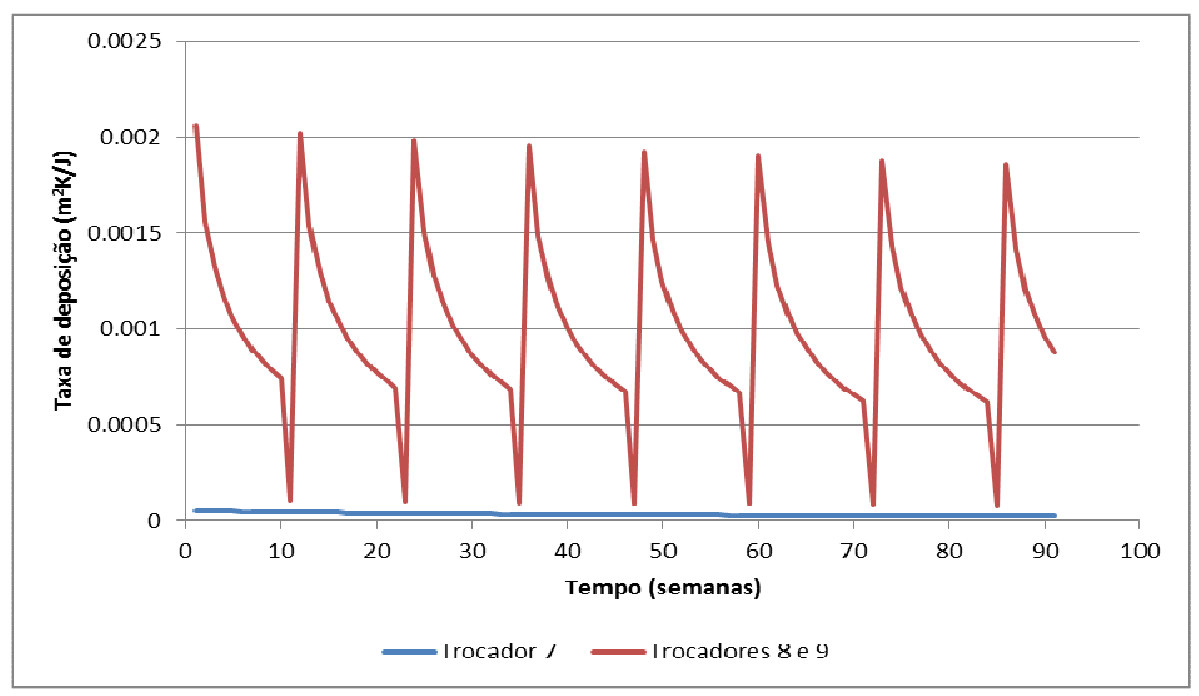

Figura 3 - Perfil da taxa de deposição ao longo do tempo

Nesta figura, é possível perceber diversos aspectos associados ao comportamento fenomenológico da deposição, que não são captados pelo modelo puramente linear: (1) Os trocadores mais próximos ao final da rede sujam mais rapidamente, pois a corrente fria está sujeita a temperaturas maiores; (2) A taxa de deposição cai com o tempo caso não haja limpezas, pois a temperatura da parede influencia diretamente a taxa, e esta decresce com o aumento da camada de depósito. Isso pode ser observado em todos os trocadores, porém é mais evidente nos trocadores do final da rede devido às suas maiores temperaturas de operação; (3) Quando um trocador de calor retorna à operação após a limpeza, sua temperatura aumenta e a taxa de deposição volta a crescer; (4) Quando um trocador retorna limpo à operação, percebe-se um leve aumento na taxa de deposição do trocador seguinte, isso pode ser explicado pelo fato da temperatura de entrada deste trocador ter 


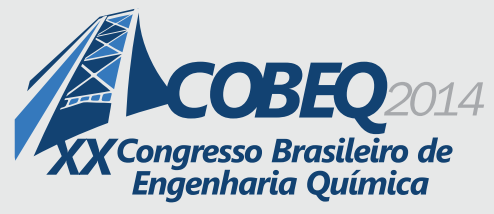

aumentado devido ao aumento na temperatura de saída do trocador anterior, com isso a temperatura de operação aumenta, aumentando a taxa de deposição.

A Figura 4 mostra o perfil da temperatura de saída da rede no caso otimizado e no base. Embora no caso otimizado a temperatura desça consideravelmente em alguns instantes, devido às retiradas para limpeza, o resultado apresenta um ganho de quase $43 \%$ em relação ao caso base, onde não há retirada de nenhum trocador para limpeza.

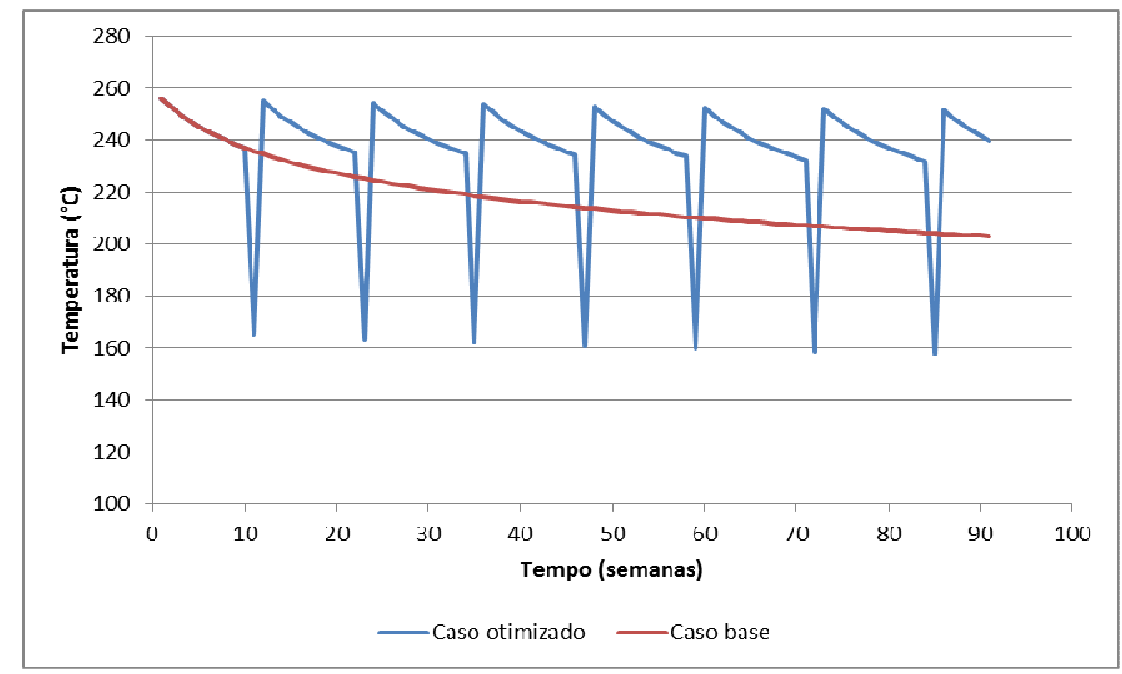

Figura 4 - Perfil de temperatura de saída da rede

\section{CONCLUSÕES}

A abordagem proposta mostrou-se eficiente na solução do problema proposto, o ganho apresentado é significativo e indica que a realização periódica de limpezas, de acordo com uma programação ótima, pode melhorar o desempenho da uma bateria de pré-aquecimento frente à deposição ao longo do tempo. Um aspecto fundamental do trabalho é evidenciar a importância da utilização de modelos fenomenológicos, pois apesar da sua maior complexidade, os resultados obtidos foram superiores aos obtidos utilizando-se um modelo linear convencional.

\section{REFERÊNCIAS}

ASSIS, B. C. G., LEMOS, J. C., QUEIROZ, E. M., PESSOA, F. L. P., LIPORACE, F. S., OLIVEIRA, S. G., COSTA, A. L. H., Optimal allocation of cleanings in heat exchanger networks. App. Therm. Eng., v. 58, p. 605-614, 2013.

LAVAJA, J. H., BAGAJEWICZ, M. J., On a new MILP model for the planning of heat exchanger network cleaning. Ind. Eng. Chem. Res., v. 43, p. 3924-3938, 2004.

LEMOS, J. C., COSTA, A. L. H., ASSIS, B. C. G., QUEIROZ, E. M., PESSOA, F. L. P., LIPORACE, F. S., OLIVEIRA, A sliding mixed-integer linear programming approach for the 
optimization of the cleaning schedule of crude preheat trains. In: Heat exchanger fouling and cleaning conference, 2013.

PANCHAL, C.B.; KURU, W.C.; LIAO, C.F.; EBERT, W.A.; PALEN, J. W. Threshold Conditions for Crude Oil Fouling. In: International Conference on Understanding Heat exchanger Fouling and its Mitigation, 1997.

SMAÏLI, F., VASSILIADIS, V.S., WILSON, D.I., Mitigation of Fouling in Refinery Heat Exchanger Networks by Optimal Management of Cleaning. Energy \& Fuels, v. 15, p. 1038-1056, 2001. 